\title{
COMMENT
}

\section{The Role of the CAG Repeat Polymorphism in the Androgen Receptor Gene and of Skewed X-Chromosome Inactivation, in the Pathogenesis of Hirsutism*}

\author{
ROSA M. CALVO, MIRYAM ASUNCIÓN, JOSÉ SANCHO, JOSÉ L. SAN MILLÁN, \\ AND HÉCTOR F. ESCOBAR-MORREALE \\ Departments of Endocrinology (R.M.C., M.A., J.S., H.F.E.-M.) and Molecular Genetics (J.L.S.M.), \\ Hospital Ramón y Cajal, 28034 Madrid, Spain
}

\begin{abstract}
The human androgen receptor (AR) gene contains a variable number of CAG repeats within exon 1. Shorter AR alleles, by increasing transactivation, may result in augmented AR-mediated sensitivity of the hair follicle. We have evaluated whether the number of CAG repeats, or if skewed inactivation of X-chromosome, favoring the presence of shorter AR alleles, influence hirsutism in women with and without hyperandrogenemia. Twenty-eight women with idiopathic hirsutism (normal serum androgens), 34 women with hyperandrogenic hirsutism (increased serum androgens), and 15 healthy control women were analyzed by evaluating the number of CAG repeats in genomic DNA, as well as the methylation pattern (after DNA digestion with HpaII), which allows identification of which allele is inactive. Despite marked differences among the groups in serum
\end{abstract}

androgens, which were markedly increased in women with hyperandrogenic hirsutism as compared with women with idiopathic hirsutism and to controls, there were no significant differences among the groups in the number of CAG repeats. Moreover, skewed X-chromosome inactivation was found in 10 subjects ( 3 with idiopathic hirsutism, 5 with hyperandrogenic hirsutism, and 2 controls; $P=0.746$ ) of the 67 women $(14.9 \%)$ who were heterozygous for the AR gene. In several of these subjects, it was the shorter allele that was preferentially inactivated.

In conclusion, neither the CAG repeat polymorphism in the AR gene, nor skewed X-chromosome inactivation, seem to play a significant role in the pathogenesis of hirsutism. (J Clin Endocrinol Metab 85: $1735-1740,2000)$
$\mathrm{H}$ IRSUTISM is considered to be a consequence of increased androgen action at the pilosebaceous unit. Most hirsute patients have abnormalities in the adrenal and/or ovarian steroidogenesis, resulting in serum androgen excess, which is responsible for their hirsutism $(1,2)$.

However, a significant proportion of hirsute patients have normal serum androgen concentrations, a condition usually termed as idiopathic hirsutism (3). The pathogenesis of hirsutism in these women is not known but might be related to a putative increase in the sensitivity of the hair follicle to androgens, resulting from abnormalities in the androgen signal transduction system in the skin.

The androgen receptor (AR) is a member of the steroid receptor superfamily and is encoded in the $\mathrm{X}$-chromosome $(4,5)$. The AR gene contains, within exon 1 , a polymorphic CAG repeat sequence, which encodes for a polyglutamine chain of variable length in the aminoterminal transactivation domain of the protein (6). The expansion of the CAG repeats

Received September 7, 1999. Revision received December 3, 1999. Accepted December 30, 1999.

Address correspondence and requests for reprints to: Héctor F. Escobar-Morreale, M.D., Ph.D., Department of Endocrinology, Hospital Ramón y Cajal, Carretera de Colmenar km. 9,100, 28034 Madrid, Spain. E-mail: hector.escobar@uam.es.

* Supported by a grant (Proyecto 08.6/0022/1998) from the Consejería de Investigación y Cultura, Comunidad de Madrid, Spain, and by grants (FIS 00/0414 and FIS 98/3044) from the Fondo de Investigación Sanitarias, Ministerio de Sanidad y Consumo, Spain. length results in a linear decrease in the transactivation function of the AR (6), and inverse relationships have been described between the number of CAG triplets in the AR gene with the risk for prostate cancer and its severity $(7,8)$, and with the hirsutism scores in Hispanic females (9). Moreover, shorter AR gene alleles have been described in association with androgenic disorders of the skin in men and women, including acne, androgenetic alopecia, and hirsutism, as compared with healthy controls (10).

Recently, skewed X-chromosome inactivation has been proposed by Vottero et al. (11) to play a role in the hypothetical hypersensitivity of the AR to androgens in women with idiopathic hirsutism. Methylation of HpaII and HhaI sites near the CAG triplets in exon 1 of the AR gene correlates with X-chromosome inactivation (12). From the study of the AR gene methylation pattern in 16 nonhyperandrogenic hirsute patients and 10 healthy control women, Vottero et al. (11) concluded that skewed inactivation of the longer of the two AR alleles, allowing for the shorter (and presumably more functional) AR allele to be expressed on the active X-chromosome, might explain the peripheral hypersensitivity to androgens present in these women.

As will be seen, after studying a large series of women diagnosed with idiopathic (nonhyperandrogenic) hirsutism or with hyperandrogenic hirsutism, as compared with healthy women, we have found that the polymorphic CAG repeat in exon 1 of the AR gene and skewed X-chromosome 
inactivation seem to play no significant role in the pathogenesis of hirsutism.

\section{Subjects and Methods}

\section{Subjects}

Sixty-two unselected hirsute women [age, $23.1 \pm$ (SD) 6.9 yr; body mass index (BMI), $25.36 \pm(\mathrm{sD}) 6.1 \mathrm{~kg} / \mathrm{m}^{2}$ ] were recruited for the study. Most of the patients did not have a family history of hyperandrogenic disorders. Hirsutism was defined by the presence of terminal hair in an androgen-dependent pattern, with a Ferriman-Gallwey score of 8 or more (13). None of the patients had hypertension, features of Cushing's disease, or drug-induced hirsutism. Hyperprolactinemia, thyroid disease, congenital adrenal hyperplasia, and acromegaly were ruled out by appropriate testing.

The reference values for the analytical procedures were obtained from a control group of 15 normal menstruating women [age, $29.5 \pm$ (SD) 8.1 yr; matched for BMI, $23.7 \pm$ (SD) $4.2 \mathrm{~kg} / \mathrm{m}^{2}$ ] who did not have signs and symptoms of hyperandrogenism or a family history of endocrine diseases. None of the patients or controls had taken hormonal medications, including contraceptive pills, for the last 6 months. All the patients and controls were Caucasians, living in Madrid, Spain. This study was approved by the hospital ethics committee, and informed consent was obtained from each patient and control.

\section{Functional evaluation}

Studies were performed between days 5 and 10 of the menstrual cycle, or during amenorrhea, after excluding pregnancy by appropriate testing. The patients reported to the Endocrine-Metabolic testing room between $0800 \mathrm{~h}$ and $0900 \mathrm{~h}$ after a 12-h overnight fast. An indwelling iv line was placed in a forearm vein, and, after 15-30 $\mathrm{min}$, basal blood samples were obtained for measurement of total testosterone (T), dehydroepiandrosterone-sulfate (DHEAS), LH, FSH, estradiol $\left(\mathrm{E}_{2}\right)$, sex hormone-binding globulin (SHBG), cortisol (F), 11-deoxycortisol (S), progesterone (P4), 17-hydroxyprogesterone (17-OHP), and $\triangle^{4}$-androstenedione (A). All blood samples were immediately centrifuged, and serum was separated and frozen at $-20 \mathrm{C}$ until assayed.

Women were considered to have idiopathic (nonhyperandrogenic) hirsutism $(n=28)$ when the basal serum concentrations of T, DHEAS, and A were within the normal range derived from the control group of 15 healthy women. The upper limits of normality for serum androgens were $2.46 \mathrm{nmol} / \mathrm{L}, 10.8 \mu \mathrm{mol} / \mathrm{L}$, and $14.0 \mathrm{nmol} / \mathrm{L}$, for T, DHEAS, and A, respectively. Women with increased concentrations of any of these androgens were considered to have hyperandrogenic hirsutism $(n=34)$.

Part of the hormonal data from 40 patients and 13 control women have been included in previous reports $(1,2,14-17)$, regarding aspects of the pathophysiology of functional hyperandrogenism different from those studied here.

\section{Hormone assays}

Serum T, LH, FSH, E2, SHBG, F, S, 17-OHP, P4, DHEAS, and A were measured as published previously $(1,2,14)$. The free androgen index was calculated using the formula $[\mathrm{T}(\mathrm{nmol} / \mathrm{L}) \times 100] /$ SHBG $(\mathrm{nmol} / \mathrm{L})]$.

\section{Determination of the number of CAG repeats in exon 1 of the $A R$}

In addition to serum samples, whole blood samples were collected from all women. Genomic DNA was extracted from peripheral leukocytes following the manufacturer's instructions (Wizard Genomic DNA Purification Kit, Promega Corp., Madison, WI; and Nucleon BAC C3, Amersham-Life Science, Buckinghamshire, UK). DNA from all subjects in the study was used to amplify the polymorphic (CAG) repeat of the human AR. PCR mixtures contained $10 \mathrm{~mm}$ Tris (pH 8.3), $50 \mathrm{~mm} \mathrm{KCl}$, $1.5 \mathrm{~mm} \mathrm{MgCl}, 200 \mu \mathrm{M}$ dNTP, 20 pmol of each primer, and $1 \mathrm{U}$ Taq polymerase (PE Applied Biosystems, Foster City, CA). The primers used for the amplification were 5'-TCCAGAATCTGTTCCAGAGCGTGC-3' labeled with TET fluorescent dye and 5'-GCTGTGAAGGTTGCTGTTCCTCAT-3' (12). Amplifications were performed by using an automated thermocycler (GeneAmp PCR System 2400; PE Applied Biosystems) applying the following conditions: an initial denaturating step at $94 \mathrm{C}$ for $5 \mathrm{~min}, 32$ cycles at $94 \mathrm{C}$ for $1 \mathrm{~min}$, at $58 \mathrm{C}$ for $1 \mathrm{~min}$, and at $72 \mathrm{C}$ for $1 \mathrm{~min}$, and a final extension at $72 \mathrm{C}$ for $7 \mathrm{~min}$. PCR products were individually diluted, and 1-2 $\mu \mathrm{L}$ were mixed with deionized formamide and a fluorescent molecular weight marker (PE Applied Biosystems). After denaturation for 5 min at $96 \mathrm{C}$, each sample was analyzed by capillary electrophoresis on an ABI 310A automated sequencer, and the PCR products were analyzed by GeneScan software (PE Applied Biosystems). The number of CAG repeats was calculated from the size of the PCR products, in relation to a series of standards obtained by direct sequencing of PCR products from five homozygous individuals.

\section{$X$-chromosome inactivation analysis}

The X-chromosome inactivation assay based on the AR gene methylation pattern using lymphocyte DNA has been described previously (12). HpaII digests only the unmethylated DNA. Methylation of HpaII site close to the (CAG)n repeat correlates with $X$ inactivation. The site is methylated on the inactive $\mathrm{X}$-chromosome and, therefore, resists cleavage by HpaII, and a PCR product is obtained from the inactive X-chromosome only.

We have used the technique modified by Pegoraro et al. (18) for use on an ABI 310 automated sequencer. In brief, for each DNA sample two reactions were prepared; in one, $1 \mu \mathrm{g}$ DNA sample was digested with $20 \mathrm{U} \mathrm{HpaII}$ at $37 \mathrm{C}$ overnight. A second aliquot with another microgram of the same DNA containing the enzyme digestion buffer but no enzyme was incubated in parallel. Reactions were stopped by incubation at 96 $\mathrm{C}$ for $5 \mathrm{~min}$. Both digested and undigested DNA (200 ng) were used for PCR amplifications. Primers and conditions were as above. All samples were amplified and analyzed, at least in duplicate, and, for each individual, the mean of these measurements was used for statistical analysis.

To quantitate the relative inactivation of AR alleles, the area of the peaks of both alleles using undigested and digested DNA were obtained using the GeneScan software. For each patient the ratio between the areas of the two alleles using undigested PCR product was calculated and used as correction factor. This correction factor was applied to compensate for unequal amplification of alleles, using digested PCR product. The degree of $\mathrm{X}$-chromosome inactivation was then calculated by normalizing the sum of both alleles to $100 \%$, and each allele was then expressed as percentage. For example, if all the cells have the same inactive X-chromosome, only one PCR product will be obtained and the result would be $100 \%$, whereas if X-chromosome inactivation is completely random, the result would be $50 \%$, indicating an equal number of cells having each of the two alleles. As discussed previously by Naumova et al. (19), an $80 \%$ or higher percentage for one allele is considered as indicative of skewed X-chromosome inactivation.

\section{Power calculations, sample size, and statistical analysis}

The power of the study to evaluate differences in the mean number of CAG repeats in the AR gene among the 28 women with idiopathic hirsutism, compared with the 34 women with hyperandrogenic hirsutism, was found to be 0.82 for the detection of differences in the means of both groups of two or more CAG repeats $(\alpha=0.05$, one-sided test, normal distribution, variance derived from the study population) (20).

Vottero et al. (11) have reported that $69 \%$ of the patients with nonhyperadrogenic hirsutism show skewed X-chromosome inactivation. Assuming that, for skewed X-chromosome inactivation to play a significant role in the pathogenesis of idiopathic hirsutism, it must be present in at least $50 \%$ of these women and that skewed X-chromosome inactivation should be found in women with hyperandrogenic hirsutism in the same proportion present in healthy women [ $14 \%$ of 64 consecutive unrelated healthy women (19)], to detect this $35 \%$ difference in the proportion of women showing skewed X-chromosome inactivation, with a power of 0.80 , the least significant number of subjects in each group must be 22 [ $\alpha=0.05$, one-sided test, normal distribution (21)]. Thus, the number of patients studied here was adequate to evaluate clinically significant differences in the number of CAG repeats and in the proportion of subjects showing skewed X-chromosome inactivation, among patients with idiopathic hirsutism and women with hyperandrogenic hirsutism.

The results are expressed as mean (median) \pm SD in the text and tables. The Kruskall-Wallis nonparametric one-way ANOVA was used to com- 
TABLE 1. Clinical and hormonal characteristics in women with idiopathic hirsutism and women with hyperandrogenic hirsutism, as compared with healthy controls

\begin{tabular}{lcrr}
\hline & Idiopathic hirsutism $(\mathrm{n}=28)$ & Hyperandrogenic hirsutism $(\mathrm{n}=34)$ & Controls $(\mathrm{n}=15)$ \\
\hline Age $(\mathrm{yr})$ & $24.8(22.5) \pm 8.2$ & $21.7(20.5) \pm 5.4^{a}$ & $29.5(28.0) \pm 8.1$ \\
BMI $\left(\mathrm{kg} / \mathrm{m}^{2}\right)$ & $24.6(24.4) \pm 4.6$ & $26.0(23.6) \pm 7.2$ & $23.7(23.4) \pm 4.2$ \\
Total T (nmol/L) & $1.76(1.77) \pm 0.5$ & $3.01(2.77) \pm 1.0^{b}$ & $1.49(1.52) \pm 0.5$ \\
DHEAS $(\mu \mathrm{mol} / \mathrm{L})$ & $6.4(6.1) \pm 2.4$ & $10.3(10.1) \pm 4.9^{b}$ & $5.3(5.0) \pm 2.8$ \\
Androstenedione (nmol/L) & $9.8(9.7) \pm 2.5$ & $14.1(13.6) \pm 6.2^{b}$ & $8.6(8.4) \pm 2.7$ \\
Free androgen index & $4.85(4.13) \pm 2.80^{a}$ & $11.57(6.97) \pm 14.66^{b}$ & $1.98(2.17) \pm 0.88$ \\
SHBG (nmol/L) & $41(33) \pm 23^{a}$ & $42(35) \pm 24^{b}$ & $81(77) \pm 26$ \\
F (nmol/L) & $432(410) \pm 176$ & $476(490) \pm 187$ & $454(440) \pm 140$ \\
S (nmol/L) & $6.6(5.8) \pm 3.2$ & $10.7(9.2) \pm 6.9^{c}$ & $6.4(6.4) \pm 3.2$ \\
17-OHP (nmol/L) & $3.1(2.5) \pm 1.9$ & $4.2(3.8) \pm 2.5^{b}$ & $2.5(2.3) \pm 1.2$ \\
P4 (nmol/L) & $3.0(3.0) \pm 1.2$ & $3.4(3.5) \pm 1.4$ & $2.6(2.4) \pm 0.8$ \\
LH (IU/L) & $3.8(3.2) \pm 2.3$ & $5.7(4.6) \pm 3.7^{c}$ & $3.9(3.6) \pm 1.6$ \\
FSH (IU/L) & $4.8(4.2) \pm 2.6$ & $5.2(4.8) \pm 2.5$ & $5.1(5.3) \pm 1.8$ \\
$\mathrm{E}_{2}(\mathrm{pmol} / \mathrm{L})$ & $169(106) \pm 125$ & $154(128) \pm 84$ & $206(132) \pm 165$ \\
CAG repeats (shorter allele) & $15(17) \pm 3$ & $15(16) \pm 3$ & $15(16) \pm 3$ \\
CAG repeats (larger allele) & $19(19) \pm 2$ & $19(19) \pm 4$ & $19(19) \pm 4$ \\
CAG repeats (both alleles) ${ }^{d}$ & $17(16) \pm 4$ & $17(17) \pm 3$ & $17(17) \pm 3$ \\
\hline
\end{tabular}

Values are means (median) \pm SD.

${ }^{a}$ At least $P<0.05$ as compared with the control group.

${ }^{b}$ At least $P<0.05$ as compared with the control group and the group of women with idiopathic hirsutism.

${ }^{c}$ At least $P<0.05$ as compared with the group of women with idiopathic hirsutism.

${ }^{d}$ Both alleles for each individual were analyzed. Homozygous individuals were counted as two alleles.

pare variables among the three groups of subjects. For variables showing significant differences between the groups by the Kruskall-Wallis test, repeated Mann-Whitney U-Wilcoxon Rank Sum W tests were used to identify the differences between each pair of groups, applying an a priori downward correction to the level of significance to compensate for multiple comparisons (i.e. $P<0.017$ is needed to reach statistically significant differences, as comparisons involve three groups) (22). A $\chi^{2}$ test was used for discontinuous variables. $P<0.05$ was considered statistically significant, with the exception stated above.

\section{Results}

Clinical and hormonal characteristics in women with idiopathic hirsutism and women with hyperandrogenic hirsutism, as compared with healthy controls

The patients with hyperandrogenic hirsutism were younger than the controls, whereas the women with idiopathic hirsutism had intermediate age that was not different from any of the other groups (Table 1). There were no differences in the BMI among the groups (Table 1), and there was no difference in the Ferriman-Gallwey score among the women with idiopathic hirsutism as compared with women with hyperandrogenic hirsutism $[15$ (13) \pm 5 vs. 17 (15) \pm 6 , $P=0.095]$.

As expected, serum T, DHEAS, and A levels and the free androgen index were higher in women with hyperandrogenic hirsutism as compared with women with idiopathic hirsutism and to controls (Table 1). Women with hyperandrogenic hirsutism also had increased serum 17-OHP levels, as compared with controls, and increased LH and S levels, as compared with women with idiopathic hirsutism (Table 1).

Finally, women with idiopathic hirsutism had increased free androgen index as compared with controls (Table 1), and SHBG levels were decreased in both groups of hirsute women as compared with controls (Table 1). There were no differences among the three groups in F, P4, FSH, and $\mathrm{E}_{2}$ circulating levels (Table 1).

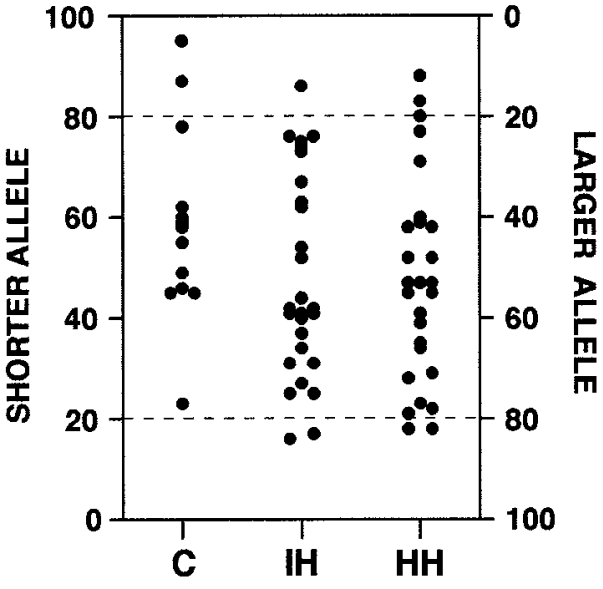

Fig. 1. Degree of X-chromosome inactivation, as assesed by methylation analysis using DNA extracted from blood leucocytes, in hirsute and control women heterozygous for the AR gene. The percentage represents the amount of total PCR product obtained from methylated, inactive DNA. Values more than $80 \%$ indicate skewed X-chromosome inactivation of the corresponding allele. C, Control group of healthy women, $\mathrm{n}=13$; $\mathrm{IH}$, idiopathic hirsutism, $\mathrm{n}=27$; $\mathrm{HH}$, hyperandrogenic hirsutism, $\mathrm{n}=27$.

\section{CAG microsatellite analysis}

There were no differences in the number of repeats among the three groups of subjects, whether analyzing all the alleles together or the smaller and larger alleles separately (Table 1). Furthermore, there were no correlations between the mean number of repeats of the pair of alleles of each subject and the Ferriman-Gallwey score or the hormonal levels (data not shown).

\section{$X$-chromosome inactivation analysis}

Ten subjects were homozygous for the AR gene, having the same number of CAG repeats in both alleles, and, there- 
TABLE 2. X-chromosome inactivation, in women with idiopathic hirsutism as compared with women with hyperandrogenic hirsutism and with healthy controls

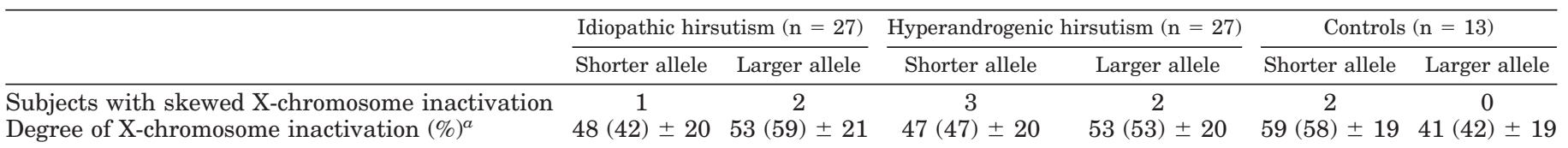

Values are means (median) \pm SD. Only women found to be heterozygous for the AR gene were analyzed. No statistically significant differences were found among the groups in any of the variables.

${ }^{a}$ HpaII digests only unmethylated, active, DNA. Therefore, the percentage for each allele of PCR product using digested DNA is inversely proportional to the number of cells having it as the active allele (i.e. an individual with $80 \%$ of the PCR product coming from the larger allele means that $80 \%$ of the cells had the larger allele methylated, and, therefore, inactive, and conversely, $80 \%$ cells have the shorter allele as the active allele).

fore, X-chromosome inactivation could not be assessed in their samples. These subjects were two controls, one patient with idiopathic hirsutism, and seven patients with hyperandrogenic hirsutism.

The remaining 67 subjects had different CAG repeats in each allele of the AR gene, but there were no differences among the groups of patients with idiopathic or hyperandrogenic hirsutism and controls, in the number of subjects with skewed X-chromosome inactivation, which was present in 10 of these 67 women (14.9\%): 3 from the group with idiopathic hirsutism, 5 from the group of hyperandrogenic hirsutism, and 2 controls (Pearson's $\chi^{2}=0.59, P=0.746$; Fig. 1 and Table 2).

It should also be noted that in one woman with idiopathic hirsutism, three patients with hyperandrogenic hirsutism, and in two control women, it was the shorter allele that was preferentially inactivated (Fig. 1 and Table 2). Furthermore, also when considering moderate degrees of skewed X-chromosome inactivation [i.e. between $65-79 \%$ (23)], there were no differences among the groups in the number of subjects affected (Pearson's $\chi^{2}=7.69, P=0.464$ ).

\section{Discussion}

Hirsutism is a consequence of increased androgen action at the pilosebaceous unit. Although most hirsute patients present increased serum androgens (3), some patients have normal levels of the main androgens, and their hirsutism is usually termed "idiopathic."

The pathophysiologic mechanisms leading to idiopathic hirsutism are not entirely known. We have previously shown that most patients with idiopathic hirsutism actually have mild adrenal and/or ovarian steroidogenic abnormalities (2).

On the contrary, other authors hypothesize that idiopathic hirsutism may result from abnormalities occurring locally at the skin. An increased activity of the enzyme $5 \alpha$-reductase, which is responsible for the conversion of $\mathrm{T}$ into the much more active dihydrotestosterone, has been proposed as the cause of idiopathic hirsutism (24-26), but a genetic basis for this hypothesis has not been demonstrated.

A second possibility is that the AR at the pilosebaceous unit of women with idiopathic hirsutism are more sensitive to their normal levels of circulating androgens than those from nonhirsute women.

As the binding of androgens to the AR is not increased in hirsute patients (25), other explanations are needed to justify this increased sensitivity of their AR. The AR is a protein that contains a polyglutamine chain in its N-terminal domain, in which the transactivation activity of the AR resides (6). The polyglutamine chain is encoded by a variable number of CAG repeats located in exon 1 of the AR gene. The expansion of the CAG repeats length decreases the transactivation of the AR in vitro (6), and, as stated above, inverse relationships have been described between the number of CAG triplets in the AR gene with the risk for prostate cancer and its severity $(7,8)$.

Recently, an inverse correlation between the number of CAG triplets in the AR gene and the hirsutism scores has been reported (9). Moreover, shorter AR gene alleles has been described in association with androgenic disorders of the skin in men and women, including acne, androgenetic alopecia, and hirsutism, as compared with healthy controls (10). Taken together, these observations suggest that shorter AR alleles result in more active AR protein (11).

Our present results, on the contrary, do not support a role for shorter AR alleles, containing less CAG repetitions, in both idiopathic or hyperandrogenic hirsutism. On the one hand, the mean number of CAG repeats were not different in women diagnosed with idiopathic hirsutism as compared with patients with hyperandrogenic hirsutism and as compared with healthy women. Moreover, short alleles, containing 10 or less CAG repeats, were found in all these groups of women, in contrast to a recent report, in which these shorter alleles were found only in women with idiopathic hirsutism (11). Finally, we have not found any correlation between the number of CAG repeats and the Ferriman-Gallwey score, or any of the hormone levels of the subjects studied here.

A provocative novel hypothesis has been proposed recently by Vottero et al. (11). All human female cells undergo inactivation of one of their X-chromosomes. Because the AR gene is located in the X-chromosome, and shorter alleles may be associated with increased AR activity, skewed inactivation of larger AR alleles in the skin would result in increased AR activity (11).

Skewing of X-chromosome inactivation seems to be an inherited character linked to specific loci of the X-chromosome $(27,28)$. In most human studies, the phenotype has been transformed into a qualitative character by defining a threshold value. X-chromosome inactivation is considered to be skewed when $80 \%$ or more of the cells of one individual show the same X-chromosome active $(19,23)$.

$\mathrm{X}$-chromosome inactivation correlates with methylation of HpaII and HhaI sites near the polymorphic CAG repeats in exon 1 of the AR gene (12), because methylation is an im- 
portant mechanism in silencing gene expression during $X$ chromosome inactivation. X-chromosome inactivation is usually assessed using DNA extracted from blood mononuclear cells. Thus, the possibility exists that the X-chromosome inactivation pattern in the hair follicle may be different from that found in tissues. However, skewed X-chromosome inactivation, as assayed from lymphocyte DNA, has been shown to influence the clinical expression of X-linked recessive traits, such as dystrophinopathy (18) and myotubular myopathy (23). Therefore, the pattern of X-chromosome inactivation in blood mononuclear cells might be a useful marker of X-chromosome inactivation in tissues.

We have found skewed $X$-chromosome inactivation in 10 $(14.9 \%)$ of 67 women heterozygous for the AR gene, a proportion similar to the $14 \%$ prevalence of skewed X-chromosome inactivation found in a series of 64 unrelated control women (19). In addition, these women were not differently distributed among women with idiopathic hirsutism, women with hyperandrogenic hirsutism, and healthy controls.

In our series, only 3 of the 28 women with idiopathic hirsutism had skewed X-chromosome inactivation, and in one of these 3 patients the smaller AR allele, and not the larger allele, was preferentially inactivated, a mechanism that may lead to decreased AR activity. Furthermore, skewed $\mathrm{X}$-chromosome inactivation was also found in a similar proportion of women with hyperandrogenic hirsutism and in controls, inactivating the larger or the shorter allele depending on the subject. Therefore, skewed X-chromosome inactivation plays a minor role, if any, in the pathogenesis of hirsutism in our series, including the subgroup of hirsute patients presenting with normal levels of circulating androgens.

On the contrary, Vottero et al. (11) found skewed X-chromosome inactivation in most of the 16 women with nonhyperandrogenic hirsutism included in their study. The methods used in both studies are essentially the same (comparison of the PCR products obtained with or without prior digestion with a methylation-sensitive endonuclease), although Vottero et al. (11) have used autoradiographic measurement of the optical densities of the radioactivity-marked primers to evaluate the PCR product and we have used an automated DNA sequencer to detect the primers labeled with a fluorescent dye.

The method used to evaluate skewed X-chromosome inactivation in the present study has several advantages over the method used by Vottero et al. (11), as pointed out by other authors $(12,18)$. On the one hand, the simplicity of the method has allowed us to evaluate a much larger series of hirsute patients, with or without hyperandrogenism, and healthy controls, giving the study the statistical power to correctly conclude the absence of an association between skewed X-chromosome inactivation and hirsutism.

Moreover, according to Pegoraro et al. (18), for each subject we have introduced a correction factor to compensate for unequal amplification of alleles. Also, the $80 \%$ cut-off value used in our study to define skewed X-chromosome inactivation, which might be more strict than the 0.4 decrease in optical density used by Vottero et al. (11), is widely supported by previous studies from other groups $(19,23)$ and is based on the original description of nonrandom X-chromosome inactivation in extraembryonic tissues of the mouse (29). These factors, and also the different ethnicity of the populations studied, might have influenced the differences between our present results and those of Vottero et al. (11).

In conclusion, our present results in unselected women with idiopathic or hyperandrogenic hirsutism do not support a role for AR-mediated hypersensitivity to androgens in the pathogenesis of hirsutism, at least by a mechanism involving the CAG repeat in the AR gene, or skewed X-chromosome inactivation.

\section{Acknowledgments}

We thank Ms. Genoveva González for her technical help with hormonal evaluation of patients and controls.

\section{References}

1. Escobar-Morreale HF, Serrano-Gotarredona J, García-Robles R, Sancho J Varela C. 1997 Lack of an ovarian function influence on the increased adrenal androgen secretion present in women with functional ovarian hyperandrogenism. Fertil Steril. 67:654-662.

2. Escobar-Morreale HF, Serrano Gotarredona J, García Robles R, Sancho J Varela C. 1997 Mild adrenal and ovarian steroidogenic abnormalities in hirsute women without hyperandrogenemia: does idiopathic hirsutism exist? Metabolism. 46:902-907.

3. Azziz R, Waggoner WT, Ochoa T, Knochenhauer ES, Boots LR. 1998 Idiopathic hirsutism: an uncommon cause of hirsutism in Alabama. Fertil Steril. 70:274-278.

4. Chang CS, Kokontis J, Liao ST. 1988 Molecular cloning of human and rat complementary DNA encoding androgen receptors. Science. 240:324-326.

5. Lubahn DB, Joseph DR, Sullivan PM, Willard HF, French FS, Wilson EM. 1988 Cloning of human androgen receptor complementary DNA and localization to the X chromosome. Science. 240:327-330.

6. Chamberlain NL, Driver ED, Miesfeld RL. 1994 The length and location of CAG trinucleotide repeats in the androgen receptor $\mathrm{N}$ terminal domain affect transactivation function. Nucleic Acids Res. 22:3181-3186.

7. Schoenberg MP, Hakimi JM, Wang S, et al. 1994 Microsatellite mutation $(\mathrm{CAG} 24 \rightarrow 18)$ in the androgen receptor gene in human prostate cancer. Biochem Biophys Res Commun. 198:74-80.

8. Hakimi JM, Schoenberg MP, Rondinelli RH, Piantadosi S, Barrack ER. 1997 Androgen receptor variants with short glutamine or glycine repeats may identify unique subpopulations of men with prostate cancer. Clin Cancer Res. 3:1599-1608.

9. Legro RS, Shahbahrami B, Lobo RA, Kovacs BW. 1994 Size polymorphisms of the androgen receptor among female Hispanics and correlation with androgenic characteristics. Obstet Gynecol. 83:701-706.

10. Sawaya ME, Shalita AR. 1998 Androgen receptor polymorphisms (CAG repeat lengths) in androgenetic alopecia, hirsutism, and acne. J Cutan Med Surg. 3:9-15

11. Vottero A, Stratakis CA, Ghizzoni L, Longui CA, Karl M, Chrousos GP. 1999 Androgen receptor-mediated hypersensitivity to androgens in women with nonhyperandrogenic hirsutism: skewing of X-chromosome inactivation. J Clin Endocrinol Metab. 84:1091-1095.

12. Allen RC, Zoghbi HY, Moseley AB, Rosenblatt HM, Belmont JW. 1992 Methylation of HpaII and HhaI sites near the polymorphic CAG repeat in the human androgen-receptor gene correlates with X-chromosome inactivation. Am J Hum Genet. 51:1229-1239.

13. Ferriman D, Gallwey JD. 1961 Clinical assessment of body hair growth in women. J Clin Endocrinol Metab. 21:1440-1447.

14. Escobar-Morreale HF, Serrano-Gotarredona J, Varela C, García-Robles R, Sancho JM. 1997 Circulating leptin concentrations in women with hirsutism. Fertil Steril. 68:898-906.

15. Escobar-Morreale HF, Serrano-Gotarredona J, Avila S, Villar-Palasí J, Varela C, Sancho J. 1998 The increased circulating prostate-specific antigen concentrations in women with hirsutism do not respond to acute changes in adrenal or ovarian function. J Clin Endocrinol Metab. 83:2580-2584.

16. Escobar-Morreale HF, Serrano-Gotarredona J, García-Robles R, Varela C Sancho JM. 1998 Abnormalities in the serum insulin-like growth factor-I axis in women with hyperandrogenism. Fertil Steril. 70:1090-1100.

17. Escobar-Morreale HF, San Millán JL, Smith RR, Sancho J, Witchel SF. 1999 The presence of the 21-hydroxylase deficiency carrier status in hirsute women: phenotype-genotype correlations. Fertil Steril. 72:629-638.

18. Pegoraro E, Schimke RN, Arahata K, et al. 1994 Detection of new paternal dystrophin gene mutations in isolated cases of dystrophinopathy in females. Am J Hum Genet. 54:989-1003. 
19. Naumova AK, Plenge RM, Bird LM, et al. 1996 Heritability of X chromosome-inactivation phenotype in a large family. Am J Hum Genet. 58:1111-1119.

20. Milton JS, Tsokos JO. 1983 Statistical methods in the biological and health sciences. New York: McGraw Hill.

21. Lemeshow S, Hosmer DW, Klar J, Lwanga SK. 1990 Adequacy of sample size in health studies. Chichester, UK: World Health Organization-John Wiley \& Sons.

22. Dawson-Saunders B, Trapp RG. 1990 Basic and clinical bioestatistics. East Norwalk, CT: Appleton \& Lange.

23. Tanner SM, Orstavik KH, Kristiansen M, et al. 1999 Skewed X-inactivation in a manifesting carrier of X-linked myotubular myopathy and in her nonmanifesting carrier mother. Hum Genet. 104:249-253.

24. Mauvais-Jarvis P. 1986 Regulation of androgen receptor and $5 \alpha$-reductase in the skin of normal and hirsute women. Clin Endocrinol Metab. 15:307-317.
25. Mowszowicz I, Melanitou E, Doukani A, Wright F, Kuttenn F, Mauvais Jarvis P. 1983 Androgen binding capacity and $5 \alpha$-reductase activity in pubic skin fibroblasts from hirsute patients. J Clin Endocrinol Metab. 56:1209-1213.

26. Stewart PM, Shackleton CHL, Beastall GH, Edwards CRW. $19905 \alpha$-Reductase activity in polycystic ovary syndrome. Lancet. 335:431-433.

27. Naumova AK, Olien L, Bird LM, et al. 1998 Genetic mapping of X-linked loci involved in skewing of $\mathrm{X}$ chromosome inactivation in the human. Eur J Hum Genet. 6:552-562.

28. Plenge RM, Hendrich BD, Schwartz C, et al. 1997 A promoter mutation in the XIST gene in two unrelated families with skewed X-chromosome inactivation. Nat Genet. 17:353-356.

29. Takagi N, Sansaki M. 1975 Preferential inactivation of the paternally derived $X$ chromosome in the extraembryonic membranes of the mouse. Nature. 256 640-642. 